\title{
UCRL-TR-236263
}

LAW RENCE LIVERMORE N A TION A L LABORATORY

\section{Recommended 236U Evaluation for the next ENDL Release}

D. A. Brown, N. Summers, I. Thompson

November 6, 2007 
This document was prepared as an account of work sponsored by an agency of the United States government. Neither the United States government nor Lawrence Livermore National Security, LLC, nor any of their employees makes any warranty, expressed or implied, or assumes any legal liability or responsibility for the accuracy, completeness, or usefulness of any information, apparatus, product, or process disclosed, or represents that its use would not infringe privately owned rights. Reference herein to any specific commercial product, process, or service by trade name, trademark, manufacturer, or otherwise does not necessarily constitute or imply its endorsement, recommendation, or favoring by the United States government or Lawrence Livermore National Security, LLC. The views and opinions of authors expressed herein do not necessarily state or reflect those of the United States government or Lawrence Livermore National Security, LLC, and shall not be used for advertising or product endorsement purposes.

This work performed under the auspices of the U.S. Department of Energy by Lawrence Livermore National Laboratory under Contract DE-AC52-07NA27344. 
UCRL-TR-??????

\title{
Recommended ${ }^{236} \mathrm{U}$ Evaluation for the next ENDL Release
}

\author{
David Brown, Neil Summers, Ian Thompson *
}

September 28, 2007

\begin{abstract}
We present a review of ${ }^{236} \mathrm{U}$ evaluations and data. We recommend adopting the ENDF/B-VII.0 ${ }^{236} \mathrm{U}$ evaluation for the next release of the ENDL database.
\end{abstract}

\section{Contents}

1 Introduction

2 Resonance Data 2

3 Fission Data 2

4 Capture Cross-Section Data $\quad 3$

5 Other High-Energy Data $\quad 5$

6 Possible future work $\quad 6$

\section{Introduction}

${ }^{236} \mathrm{U}$ is the product of neutron capture on ${ }^{235} \mathrm{U}$ so it is both naturally occuring and present in various applications. It is a threshold fissioner and therefore has a large capture cross-section. Thus, it is present in significant quantities in various applications and, if one knows how much is present with some degree of fidelity, one may use it as a diagnostic for the thermal and fission spectrum neutron flux.

We compare various ${ }^{236} \mathrm{U}$ evaluations available and argue for the adoption of the ENDF/B-VII.0 evaluation. The evaluations we consider are ENDF/B-VII.0 [1], JEFF-3.1 [2], and JENDL-3.3 [3]. Here we will review some of the apparently more problematic parts of the evaluation. We will resolve some of these issues, justifying our adoption of this evaluation in the next release of the ENDL database.

${ }^{*}$ Lawrence Livermore National Laboratory, Livermore, CA 94550 USA 


\begin{tabular}{rccccc}
\hline \hline & JENDL-3.3 & JENDL-3.3 & ENDF/B-VII.0 \\
$\sigma(0.0253$ ev $)$ & $\begin{array}{c}\text { R.I. } \\
\text { (barns) }\end{array}$ & $\begin{array}{c}\sigma(0.0253 \text { ev }) \\
\text { (barns })\end{array}$ & $\begin{array}{c}\text { R.I. } \\
\text { (barns) }\end{array}$ & $\begin{array}{c}\text { Data } \\
\text { R.I. } \\
\text { (barns) }\end{array}$ \\
\hline \hline Total & 13.69 & - & - & - & - \\
Elastic & 8.337 & - & - & - & - \\
Fission & 0.0613 & 7.77 & 0.047 & 7.77 & $7.8 \pm 1.6$ \\
Capture & 5.295 & 346. & 5.13 & 338 & $360 \pm 15$ \\
\hline \hline
\end{tabular}

Table 1: Calculated thermal cross-sections and resonance integral (R.I.).

\section{Resonance Data}

There are basically two resonance region evaluations for ${ }^{236}$ U. ENDF/B-VII.0's resolved and unresolved resonance parameter sets were adopted from the Mann and Schenter evaluations of 1989 and 1978 respectively. The JEFF-3.1 and JENDL-3.3 evaluations are identical and appear to come from the T. Yoshida (NAIG) evaluation in JENDL-2.

There is little data to guide any of the evaluations. The resonance parameters in both the ENDF/B-VII.0 evaluation and the JENDL based evaluations are derived from the measurements of [4], [5], and [6]. The two sets of evaluations have slightly different negative energy resonances which provide the smooth background. In addition, ENDF/B-VII.0 one has about 10 more $(\mathrm{n}, \gamma)$ resonances. Asside from these extra resonances, the ENDF/B-VII.0 cross-sections are generally lower than those in JEFF-3.1 and JENDL-3.3. There is also fission data from the Pommard bomb-shot [7], but the data is not high enough quality to distinguish between the evaluations. Table 1 shows the thermal cross-sections and resonance integrals.

Given the state of the resonance evaluations and accompanying data, we could benefit from better ${ }^{236} \mathrm{U}(\mathrm{n}, \mathrm{f})$ and ${ }^{236} \mathrm{U}(\mathrm{n}, \gamma)$ resonance measurements. In the absense of this, both sets of evaluations are of equal quality so we adopt ENDF/B-VII.0.

\section{Fission Data}

The high energy $(<1 \mathrm{MeV})$ fission cross-section in the ENDF/B-VII.0 evaluation is a fit to data. The LANL evaluators took the large set of ${ }^{236} \mathrm{U}(\mathrm{n}, \mathrm{f}) /{ }^{235} \mathrm{U}(\mathrm{n}, \mathrm{f})$ data and converted it into absolute ${ }^{236} \mathrm{U}(\mathrm{n}, \mathrm{f})$ data using the ENDF/B-VII.0 standards evaluation of ${ }^{235} \mathrm{U}(\mathrm{n}, \mathrm{f})[8]$. In Fig. 1, we show the ratio data compared with the same ratio constructed from the ENDF/B-VII.0 evaluations. This ratio data starts at around the fission threshold and runs to roughly $20 \mathrm{MeV}$. The LANL evaluators did not include the absolute fission measurements shown in Fig. 2. Had they, they would have found excellent agreement with all of the data save the Rosler, Plasil, Schmitt data [9] and the Henkel data [10]. Neither set was published, neither contain uncertanties, and neither provide documentation; therefore they may be safely excluded from consideration. The LANL evaluators would also have found reasonable agreement with the Cramer, Bergen [11] data, after applying the 0.8 renormalization discussed by Younes and Britt [12] and Lynn and Hayes [13]. This factor is not related to the underestimation of the neptunium contamination noted also by Younes and Britt, but is rather due to some other unidentified equipment problem in the Pommard bomb shot experiment [7].

In any event, the JEFF-3.1 and JENDL-3.3 evaluations are also in excellent agreement with the fission cross-section data as well.

Both ENDF/B-VII.0 and JENDL-3.3 use the Brady and England evaluation [14] of the delayed $\bar{\nu}$ and the Malinovskii evaluation [15] for the prompt $\bar{\nu}$. ENDF/B-VII.0 carries over the fission spectrum from ENDF/B-VI.8 which is a simple Watt spectrum as is JENDL-3.3's fission spectrum. JEFF-3.1, on the other hand, adopted a much more agressive scheme. They used the Vladuca and Tudoro model [16] for the prompt $\bar{\nu}$ and the outgoing fission spectrum. The delayed $\bar{\nu}$ in JEFF-3.1 is from Ref. [17].

There is no data to guide the choice of the outgoing fission neutrons or $\bar{\nu}$ in this case. So, on one hand, there is no reason to not adopt ENDF/B-VII.0. However, JEFF-3.1 is newer and based on a more 


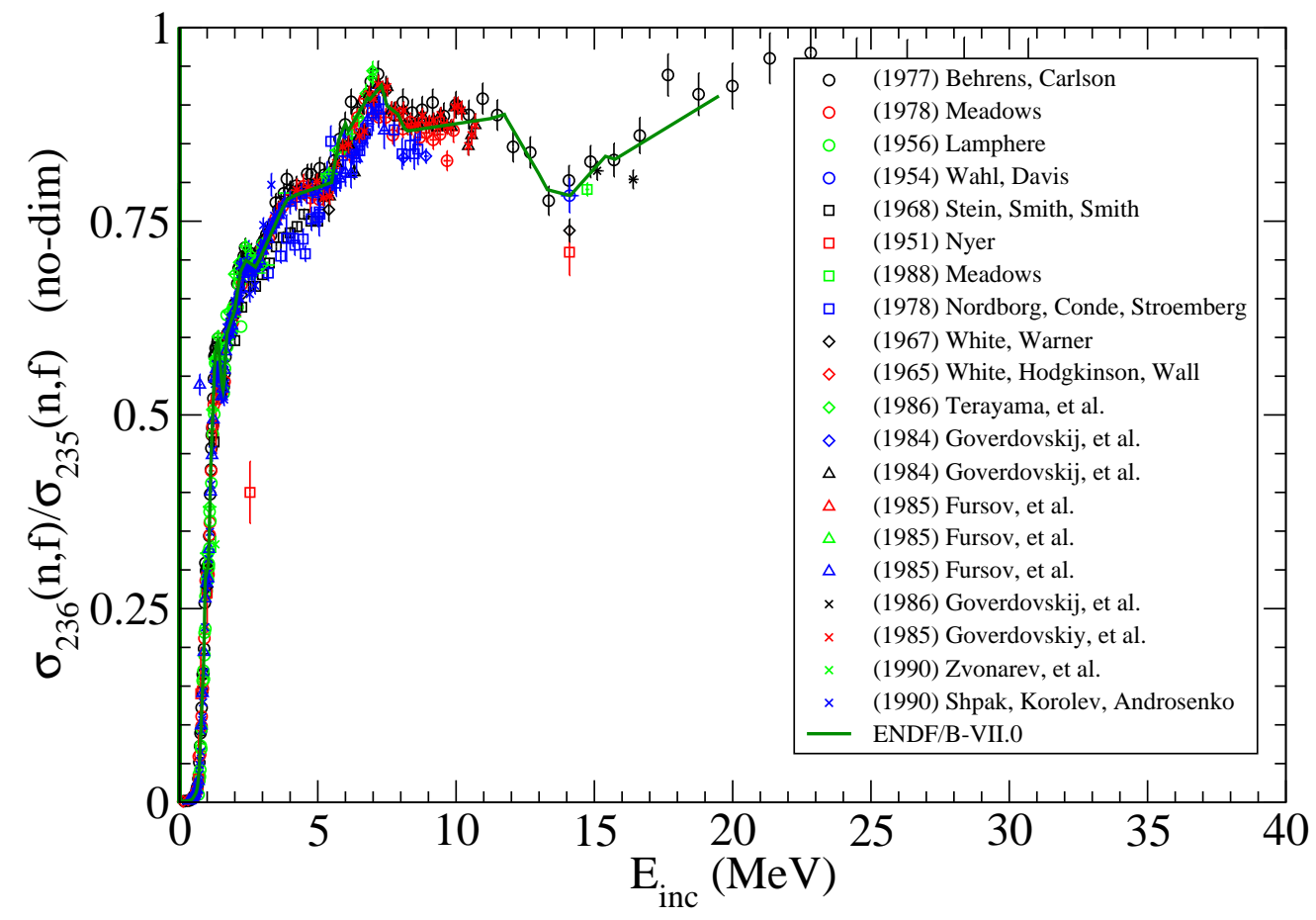

Figure 1: Ratio cross-section data for ${ }^{236} \mathrm{U}(\mathrm{n}, \mathrm{f})$, compared to the ENDF/B-VII.0 fit.

sophisticated model. Certainly the Vladuca and Tudora model and its predecessor Madland-Nix model give very reasonable fission neutron spectra for other actinides.

\section{Capture Cross-Section Data}

At high energy $(>300 \mathrm{keV}$ ), we see in Fig. 3 that there is a significant discrepancy between the various capture cross-section measurements and the evaluated data. We will try to disentangle these measurements and determine which one(s) should be used in the evaluation. Below this energy all of the data and evaluations are in agreement as one can see in Fig. 3.

All of the high energy capture measurements were both performed using activation methods. The experimentors irradiated samples enriched in ${ }^{236} \mathrm{U}$, then measured the amount of ${ }^{237} \mathrm{U}$ produced in the sample. Thus, it is important to understand the initial sample purity and mass, and the incident neutron flux as a function of irradiation time. Since ${ }^{236} \mathrm{U}$ is fissile and there are ${ }^{235} \mathrm{U}$ impurities in the sample (since it is produced via ${ }^{235} \mathrm{U}$ irradiation), the fission fragments also provide a background that must be dealt with. To measure the amount of ${ }^{237} \mathrm{U}$ produced in the experiments, the experimentors measured the activity of ${ }^{237} \mathrm{U}$ through $\beta$ decay to ${ }^{237} \mathrm{~Np}$, which produces a distinctive gamma decay spectrum. In order to provide an absolute normalization, the various experiments also measured the ${ }^{235} \mathrm{U}(\mathrm{n}, \mathrm{f})$ cross-section from a control ${ }^{235} \mathrm{U}$ foil as a monitor.

In the more recent activation experiments, ([18],[19],[20],[21]), the decay gamma spectrum is measured with $\mathrm{Ge}(\mathrm{Li})$ detectors with fine enough energy resolution to tune on the $277.64 \mathrm{keV}$ gamma line from ${ }^{237} \mathrm{~Np}$. Thus the fission fragment background is mostly eliminated since their decays produce no such gamma. A. N. Davletshin et al. [22] provide a detailed account of the experimental analysis in Ref. [18], elaborating greatly on their model of the sample activation and subsequent decay. This account gives us confidence in the results from their collaboration. The various Trofimov data sets $([19],[20],[21])$ use a very simplistic analysis, rendering those data untrustworthy.

Stupegia et al. [23] and Barry et al. [24] are old measurements which predate the general usage of Ge 


\section{${ }^{236} \mathrm{U}(\mathrm{n}$, Fission $)$}
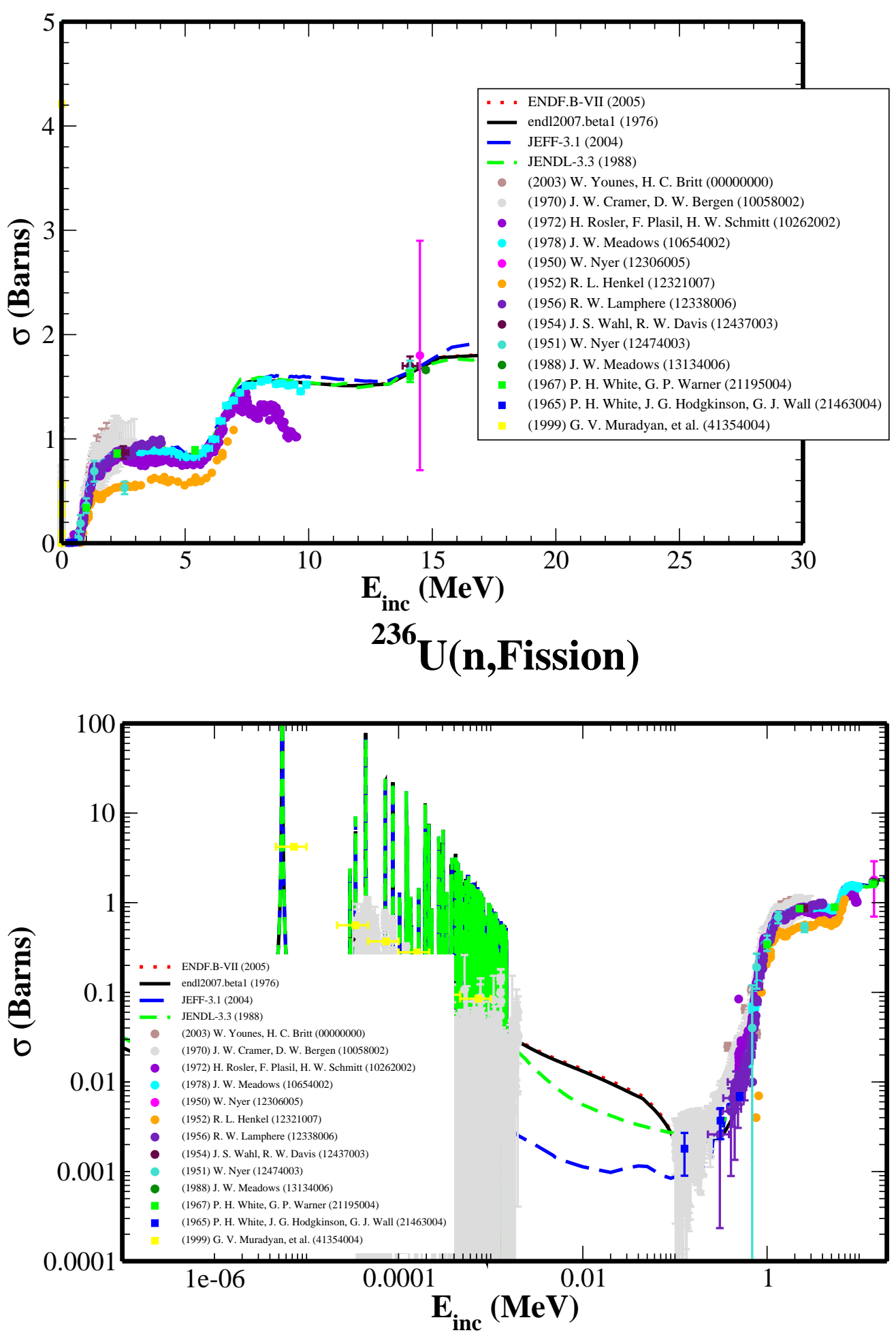

Figure 2: Absolute cross-section data for ${ }^{236} \mathrm{U}(\mathrm{n}, \mathrm{f})$. 


\section{${ }^{236} \mathrm{U}(\mathbf{n}$, gamma $)$}

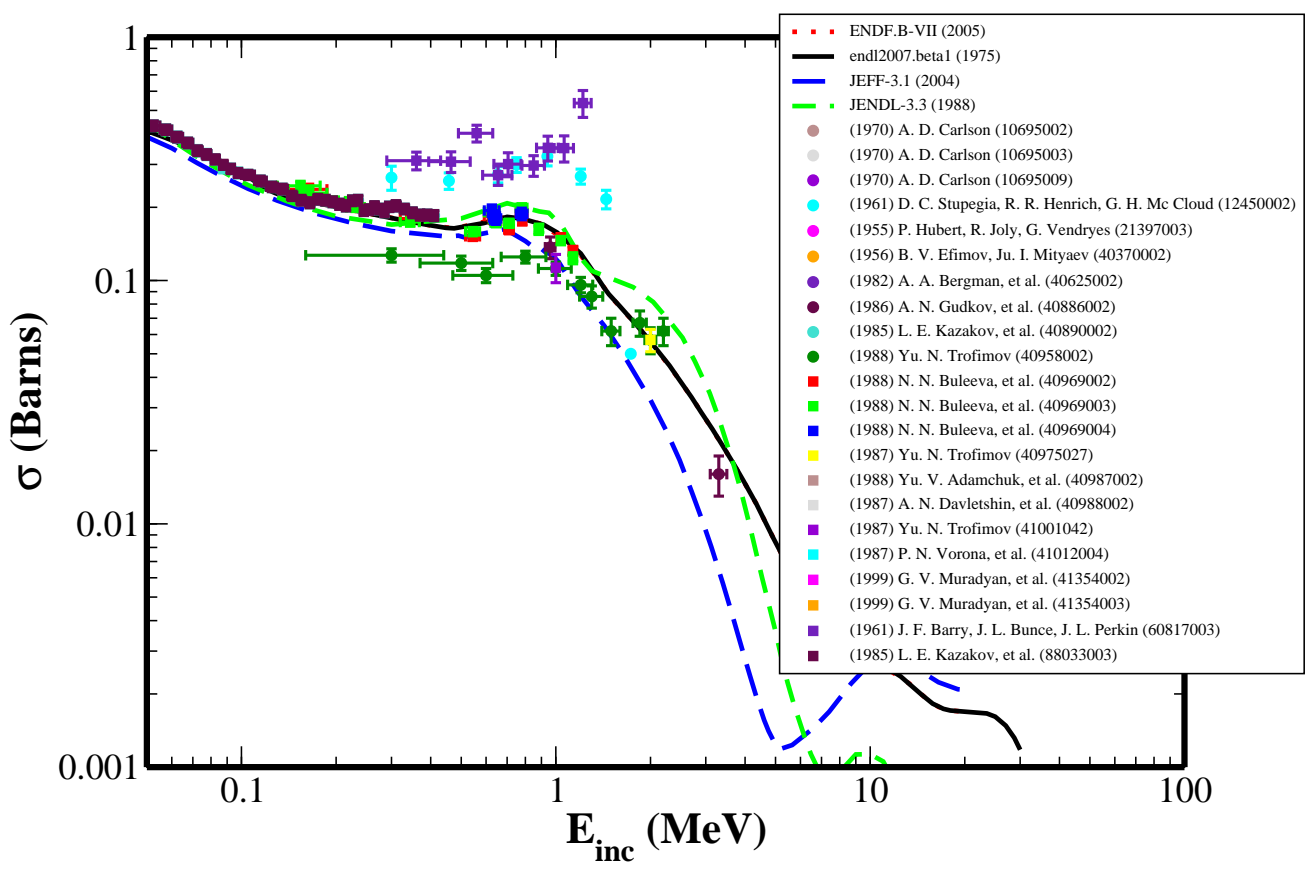

Figure 3: Plots of $(\mathrm{n}, \gamma)$ cross-section data.

detectors. Both used NaI detectors which have poor energy resolution. To deal with this problem, both experiments used complicated chemical processing to remove fission fragments from the sample to obtain pure ${ }^{237} \mathrm{U}$. To test the efficacy of this processing, they applied the same chemical processing to the ${ }^{235} \mathrm{U}$ monitor foils. The gamma activity of the foils before and after separation provided a correction factor for the ${ }^{236} \mathrm{U}$ separation. The separation process always results in some sample loss, which is not described in either experiment. The correction factor from this approach is only good provided the fission fragment distributions from ${ }^{235} \mathrm{U}$ and ${ }^{236} \mathrm{U}$ are similar enough. If all this is not enough, both experiments use a very old ${ }^{235} \mathrm{U}(\mathrm{n}, \mathrm{f})$ cross-section evaluation from Ref. [25]. When we use a modern evaluation, the discrepancy between the Stupegia et al. and Barry et al. sets and the Buleev et al. sets and the ENDF/B-VII.0 evaluation shrinks, but not enough to account for the factor of two difference. Given these questions, we feel confident in discarding these two data sets.

Now, we consider the various evaluations in light of this discussion. The JEFF-3.1 evaluation seems to take the Trofimov data seriously, going under the Buleev data. The JENDL-3.3 evaluation runs high of the Buleev et al. data, presummably because that evaluation pre-dates both the Buleev et al. and Trofimov data sets. Only the ENDF/B-VII.0 evaluation actually is in agreement with the Buleev et al. data.

\section{Other High-Energy Data}

The remainder of the high-energy cross-sections are shown in Fig. 4.

Our main concern with the high-energy cross-section data is the fact that the ENDF/B-VII.0 evaluators fit the fission cross-section and may not have adjusted the other calculations to account for the changed interchannel competiton. However, they have addressed this issue by tuning the GNASH fission cross-section to match the data as well as possible before using the other cross-section data. The other outgoing distributions also done within GNASH, so are consistent with cross-sections. 
We have no reason to suspect the same in the JEFF-3.1 and JENDL-3.3 evaluations other than they are in rough agreement with the ENDF/B-VII.0 evaluation for reactions such as the (n,2n) reaction.

\section{Possible future work}

While we recommend adopting the ENDF/B-VII.0 ${ }^{236} \mathrm{U}$ evaluation, we are curious to see how well the evaluation would perform if we replaced the outgoing fission neutron spectra and $\bar{\nu}$ with those in JEFF-3.1.

\section{Acknowledgments}

The authors would like to thank Mark Stoyer for his explanation of the radiochemistry involved in the determination of the ${ }^{236} \mathrm{U}(\mathrm{n}, \gamma)$ cross-section. This work was performed under the auspices of the U.S. Department of Energy by University of California, Lawrence Livermore National Laboratory under Contract W-7405-Eng-48.

\section{References}

[1] P.G. Young, et al., ENDF/B-VII.0 Material 9231 (2006).

[2] M-J. Lopez-Jimenez, B. Morillon, P. Romain, JEFF-3.1 Material 9231 (2004).

[3] T. Yoshida, JENDL-3.3 Material 9231 (1993).

[4] G. Carraro, et al., Nucl. Phys., A275 p. 333 (1976).

[5] L. Mewissen, et al., 1975 Washington, p. 729 (1975).

[6] J.P. Theobald, Nucl, Phys., 181, p. 637 (1972).

[7] G.A. Cowen, G.A. Jarvis, G.W. Knobeloch, B. Warren, LANL Report LA-1669, (1955); LANL Report LA-1669; LANL Report LA-4041; LANL Report LA-4095;

[8] P.G. Young, et al., ENDF/B-VII.0 Material 9228 (2006).

[9] H. Rosler, F. Plasil, H.W. Schmitt (1972), Exfor entry 10262002.

[10] R.L. Henkel (1952), Exfor entry 12321007.

[11] J.W. Cramer, D.W. Bergen (1970), Exfor entry 10058002.

[12] W. Younes, H. C. Britt, LLNL Report UCRL-TR-212600 (2005); Phys. Rev. C 68, 034610 (2003).

[13] J. E. Lynn, A. C. Hayes Phys. Rev. C 67, 014607 (2003).

[14] M.C. Brady and T.R. England, Nucl. Sci. Eng., 103 (1989) 129.

[15] V.V. Malinovskii et al., Atomnaya Energiya, 53 (1982) 83.

[16] A. Tudora, Ann. Nucl. En., 33, pp 1030-1038 (2006); G. Vladuca, A. Tudora, Comput. Phys. Commun. 125, pp. 221-238 (2000); Ann. Nucl. En. 28, pp. 1643-1652 (2001); Ann. Nucl. En. 28, pp. 419-435 (2001); Ann. Nucl. En. 28, pp. 689-700 (2001).

[17] Rugama, NEA/OECD, Jefdoc-976 (2005); Spriggs, Campbe, Piksaikin, Prog. Nucl. Eng. 41, p.223 (2002).

[18] N.N.Buleeva et al. Atomnaya Energiya,65,(6), p. 348 (1988); Exfor entry 40969002. 

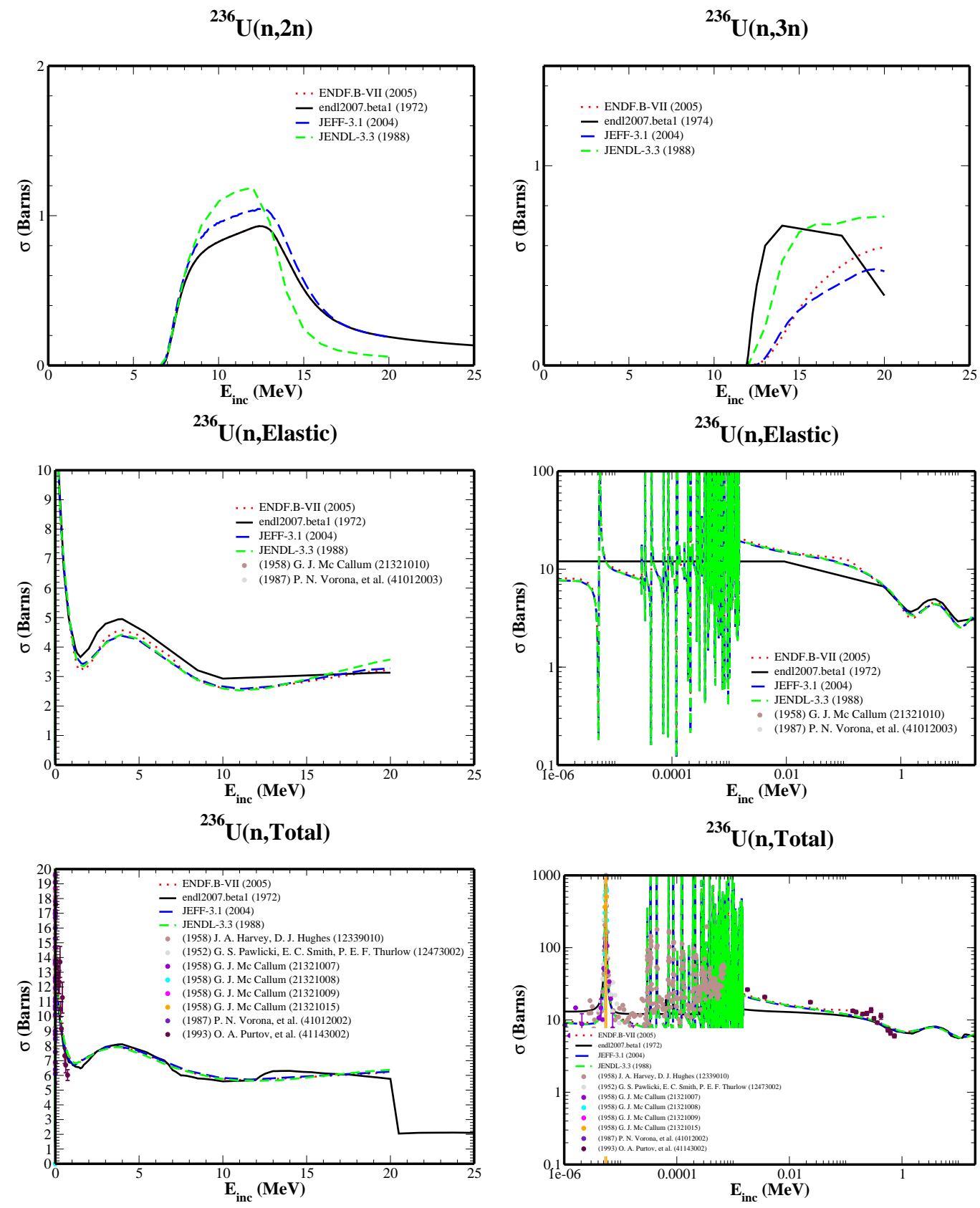

Figure 4: Plots of selected cross-section data contained in this evaluation. 
[19] Yu.N.Trofimov Atomnaya Energiya,64,(2), p. 150 (1988); Exfor entry 40958002.

[20] Yu.N.Trofimov J,YK,1987,(4),10,1987; Exfor entry 40975027.

[21] Yu.N.Trofimov Proceedings of the Conf. on Neutron Physics, 3, p. 331 (1987); Exfor entry 41001042.

[22] A. N. Davletshin et al. Atomnaya Energiya,65,(6),343-348 (1988).

[23] D.C. Stupegia et al. J. Nucl. Energy Parts A+B, 15, p. 200 (1961); Exfor entry 12450002.

[24] J.F. Barry et al. Proc. Phys. Soc., 78, p. 801 (1961)

[25] D.J. Hughes, R.B. Schwartz, U.S. Atomic Energy Commission Report, BNL 325, 2nd Ed. (Washington: US Government Printing Office) (1958); J.R. Stehn, M.D. Goldberg, R. Wiener-Chasman, S.F. Mughabghab, B.A. Magurno, V.M. May BNL-325 2nd Ed. Suppl. \#2 (Vol III, Z=88 to 98) (1965). 